\title{
Cavity-enhanced simultaneous dressing of quantum dot exciton and biexciton states
}

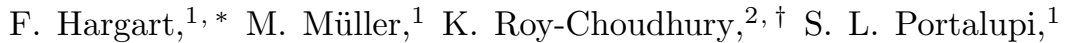 \\ C. Schneider, ${ }^{3}$ S. Hölling, ${ }^{3,4}$ M. Kamp, ${ }^{3}$ S. Hughes, ${ }^{2}$ and P. Michler ${ }^{1}$ \\ ${ }^{1}$ Institut für Halbleiteroptik und Funktionelle Grenzflächen, Research Center SCoPE and IQST, \\ Universität Stuttgart, Allmandring 3, 70569 Stuttgart, Germany \\ ${ }^{2}$ Department of Physics, Engineering Physics and Astronomy, \\ Queen's University, Kingston, Ontario, Canada K7L 3N6 \\ ${ }^{3}$ Technische Physik and Wilhelm Conrad Röntgen Research Center for Complex Material Systems, \\ Physikalisches Institut, Universität Würzburg, Am Hubland, 97074 Würzburg, Germany \\ ${ }^{4}$ SUPA, School of Physics and Astronomy, University of St. Andrews, St. Andrews, KY16 9SS, United Kingdom
}

(Dated: February 23, 2016)

\begin{abstract}
We demonstrate the simultaneous dressing of both vacuum-to-exciton and exciton-to-biexciton transitions of a single semiconductor quantum dot in a high-Q micropillar cavity, using photoluminescence spectroscopy. Resonant two-photon excitation of the biexciton is achieved by spectrally tuning the quantum dot emission with respect to the cavity mode. The cavity couples to both transitions and amplifies the Rabi-frequency of the likewise resonant continuous wave laser, driving the transitions. We observe strong-field splitting of the emission lines, which depend on the driving Rabi field amplitude and the cavity-laser detuning. A dressed state theory of a driven 4-level atom correctly predicts the distinct spectral transitions observed in the emission spectrum, and a detailed description of the emission spectra is further provided through a polaron master equation approach which accounts for cavity coupling and acoustic phonon interactions of the semiconductor medium.
\end{abstract}

PACS numbers: $78.67 . \mathrm{Hc}, 42.50 . \mathrm{Hz}, 78.55 . \mathrm{Cr}$

\section{INTRODUCTION}

Single-photon emission from a resonantly driven quantum emitter has attracted increasing attention both from fundamental science perspectives and as a potential quantum light source for applications in quantum information science. Applying a strong continuous wave (CW) laser on a two-level transition facilitates hybrid light-matter systems, typically described in a dressed-state picture ${ }^{12}$. The study of dressed states in semiconductor quantum dots (QDs) and their emission properties has become a vital research field in semiconductor quantum optics. In previous work on dressed exciton-biexciton states in QDs, either the ground stateexciton transition $(|G\rangle-|X\rangle)$ or the exciton-biexciton transition $(|X\rangle-|X X\rangle)$ were dressed by a strong coupling laser ${ }^{3}$ 10. The dressed states were studied with two-color experiment ${ }^{3 / 6}$ or with resonance fluorescence studies, i.e., observing the characteristic Mollow triplet ${ }^{7}$ 10.

Cascaded single-photon emission from the Mollow triplet sidebands of a $\mathrm{QD}^{111}$ and linewidth broadening ${ }^{12 \mid 13}$ due to excitation-induced dephasing have been demonstrated. Furthermore, the resonant coupling of a Mollow triplet sideband to an optical cavity has been studied ${ }^{14}$, a decrease of the Mollow triplet sideband splitting was observed with increasing temperature ${ }^{\sqrt{15}}$, and Mollow quintuplets from coherently excited QDs have been presented 16 . Lately, a doubly-dressed single QD exposed to a bichromatic laser field has been studied thereby observing the nearly complete elimination of the resonance fluorescence spectral line ${ }^{17}$. Systematic studies of twocolor second-order correlations of the light scattered nearresonantly by a QD have also been presented 18 . In ad- dition, two-color experiments where a second weak laser probes the dressed states generated by a strong laser field on the QD have been performed; e.g., a second probe laser was used to measure splitting of the dressed states by differential transmission ${ }^{3 / 5}$ or for excitation of excess charge carriers in the barrier to generate QD photoluminescence (PL), thus revealing dressed states ${ }^{6}$. In this context, high-Q microresonators have successfully been used to enhance the electric field of a driving laser and to demonstrate large optical Stark shifts in single QDcavity systems as well as in QD molecule spin-cavity systems, providing an all-optical method to control the spinexchange splitting 9

In this work we demonstrate, for the first time to our knowledge, simultaneous dressing of vacuum-to-exciton and exciton-to-biexciton transitions in a semiconductor QD by tuning the frequency of a single drive laser close to half a biexciton energy. Such frequency tuning allows us to establish the two-photon resonance condition for direct, coherent excitation of the biexciton state. The optically prepared biexciton state $|X X\rangle$ can spontaneously decay via the $x$ - or the $y$-polarized single exciton states $(|X\rangle$ or $|Y\rangle)$. In the current experiment the two $x$-polarized branches $(|X X\rangle-|X\rangle-|G\rangle)$ are coherently driven, and the $y$-polarized emission spectrum $S_{\mathrm{Y}}$ is measured. Calculations using a simple 4-level atom model predicts 6 spectrally distinct transitions in the $y$ polarized spectrum $S_{\mathrm{Y}}$, which allows a direct observation of the dressed states created by the $x$-polarized drive laser. Strong enhancement of the driving lasers electric field within a high-Q micropillar cavity further aids the coherent dressing. Simulations using a more complete polaron master equation approach for the QD-cavity system 


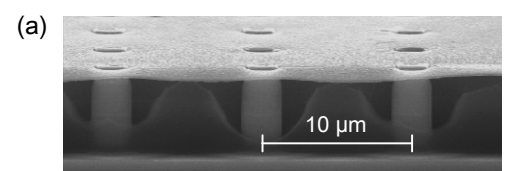

(b) Optical setup

(c) QD + cavity
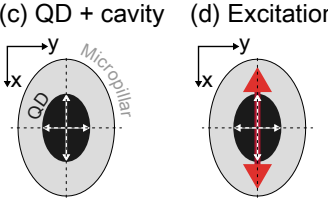

(e) Side detection
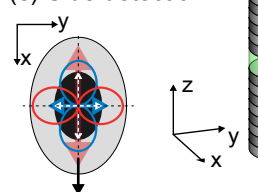

FIG. 1. (Color online) (a) Scanning electron microscope image of the sample with an Al mask on top. (b) Schematic of the optical accessibility illustrated for the resonant cavity drive. Two orthogonal polarizers are used to reduce scattered light. (c) For the system under investigation the QD and cavity axes are roughly orientated to each other. (d) Resonant excitation: The driving laser field is resonant to the x-polarized $\mathrm{FM}_{x}$ and, thus, only drives the $|X\rangle$ transitions of the QD. (e) Detection: The biexciton state $|X X\rangle$ can decay via both channels $|X\rangle$ and $|Y\rangle$ but only the $y$-polarized transitions emit into the side detection path. This is due to the dipole radiation pattern, illustrated by the colored, vertical sections of a toroid.

shows excellent agreement with the experimental measurements.

Our paper is organized as follows. In Sec. II], we introduce the sample, the optical setup and the QD-cavity system under investigation. In Sec. III we first demonstrate two-photon excitation of the biexciton state under CW pumping, enhanced by the cavity interaction. The occurrence of dressed states is then described by a simple, intuitive 4-level atom model. Thus, the reader can easily understand the demonstrated power and detuning dependencies of the dressed states. We then introduce our full polaron master equation model in Sec. IV, including cavity and phonon interactions. In Sec. V we comment on the possibility to use this unique kind of dressed states for the generation of Fock states regarding recent theoretical proposals. We present our conclusions in Sec. VI. Finally, we give an appendix where we prove the biexciton-exciton cascade via power dependent and cross-correlation measurements, and we present the complete set of dressed states for both $x$-polarized and $y$-polarized photons.

\section{METHODS}

\section{A. Sample structure and optical setup}

The sample is grown by molecular beam epitaxy and is composed of self-assembled $\operatorname{In}_{0.45} \mathrm{Ga}_{0.55}$ As QDs embedded in a high-Q micropillar resonator with a diameter of $2 \mu \mathrm{m}$. Two distributed Bragg reflectors consisting of 26 (top) and 30 (bottom) pairs of alternating $\lambda / 4$ thick layers of GaAs and AlAs form the Fabry-Pérot like $\lambda$ - cavity. A thin aluminum mask on top of the micropillars to reduce scattered laser light was fabricated by previously depositing Benzocyclobutene. The latter is then partially etched with oxide plasma. A scanning electron microscope image of the fully processed sample is displayed in Fig. 11(a).

The sample is mounted inside a helium flow cryostat accessible via two microscope objectives $(\mathrm{NA}=0.45)$ in an orthogonal configuration, which allows for excitation and detection from both the top and the side of all micropillars located close to the edge of the sample. For our studies of dressed states we later couple the resonant, x-polarized laser from the top into the cavity mode and detect the QD emission from the side (Fig. 1(b)). A pair of crossed polarizers with an extinction ratio of more than $10^{5}$ in front of the objectives further suppresses laser straylight. The QD emission is analyzed using a standard spectrometer with a $1800 \mathrm{l} / \mathrm{mm}$ grating and a CCD camera with a spectral resolution of about $28 \mu \mathrm{eV}$.

\section{B. QD-cavity system under study}

To investigate basic optical parameters of the QDcavity system we perform micro-PL spectroscopy, with the pump laser at a frequency above the GaAs band edge and an incident power of $0.11 \mu \mathrm{W}$. Using the top detection and two orthogonal positions of the polarizer, i.e., x- and y-polarization, we observe two cross-polarized fundamental modes (FMs) with an energy splitting of $320 \mu \mathrm{eV}$ and quality factors around $18500\left(\mathrm{FM}_{x}\right)$ and $10300\left(\mathrm{FM}_{y}\right)$, respectively (Fig. 2(a)). This characteristic is well-known for elliptically shaped micropillars 21 . Using the side detection, the QD emission becomes more distinctive, as the FM emission is strongly directional to the top but the QD emission is also leaking to the side (Fig. 2(b)). We observe two emission lines at roughly $1.364 \mathrm{eV}$ and $1.366 \mathrm{eV}$, attributed to a biexciton-exciton cascade by means of linear and quadratic power dependence and second-order cross-correlation measurements (see appendix A). At a temperature of $6.8 \mathrm{~K}$, these two lines are spectrally symmetric to the $x$-polarized $\mathrm{FM}_{x}$ at $1.365 \mathrm{eV}$ enabling the cavity-enhanced, coherent preparation of the biexciton state $|X X\rangle$ via two-photon absorption. Due to the low probability of this non-linear process recent works have been limited to pulsed excitation $22+28$.

\section{EXPERIMENTAL REALIZATION OF AN DRESSED 4-LEVEL SYSTEM}

\section{A. Continuous-wave two-photon excitation}

In the following experiments, we couple the resonant CW laser with frequency $\omega_{L}$ and a linewidth $<2 \mathrm{MHz}$ from the top into the $x$-polarized cavity mode with frequency $\omega_{c}^{x}$. The electric field is strongly enhanced by the cavity mode, thus, enabling the two-photon excitation 
(a)

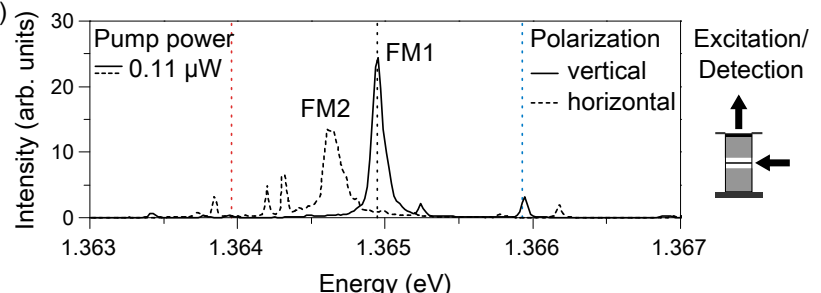

(b)

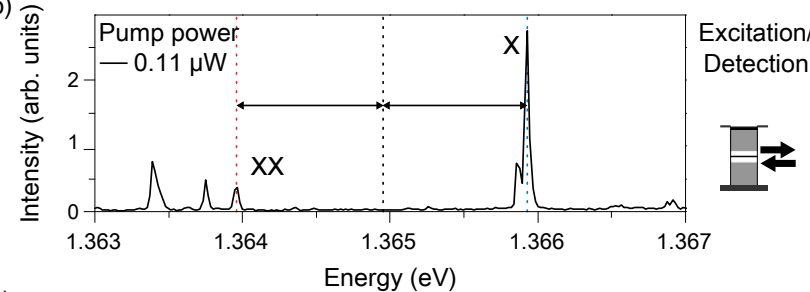

(c)

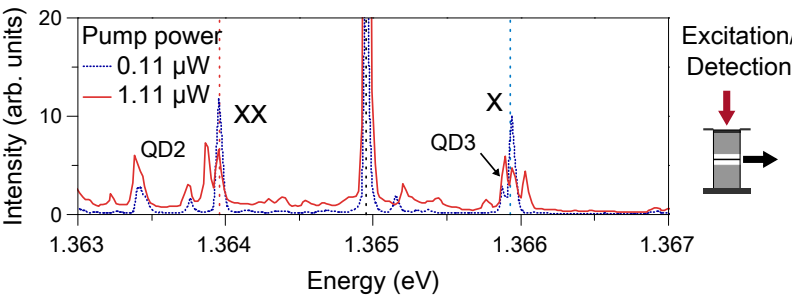

FIG. 2. (Color online) (a) Polarization resolved spectra for above bandgap excitation and top detection. (b) Side detection: The mode emission is suppressed whereas the biexciton $\mathrm{XX}$ and exciton X emission lines are observable. (c) Two spectra for resonant cavity drive. Weak pump (blue, dotted): single biexciton XX and exciton X emission lines. Strong pump (red, solid): dressed XX and X emission lines.

under $\mathrm{CW}$ excitation with low pump powers. The QD emission is collected from the side. In Fig. 2(c), we show two spectra, one for moderate pump power (blue, dotted) and one for strong pump power (red, solid). At moderate pump power, the resonant excitation of the biexciton state results in the typical biexciton-exciton cascade with almost equal intensities for both emission lines, which already serves as a hint for the direct preparation of the biexciton state. The bright signal at $1.365 \mathrm{eV}$ corresponds to scattered laser light. Several weaker emission lines are observable and might come from other QDs which are incoherently excited (typically through phonon-assisted processes). We label two lines as QD2 and QD3 to avoid confusion with the actually investigated QD.

For a tenfold increased excitation power a distinct symmetric splitting of both the biexciton and the exciton is observable. The magnitude of the splitting is $\hbar \Delta_{\Omega}=80 \mu \mathrm{eV}$. In contrast, no other lines exhibit such a splitting, instead most of them are shifted due to an AC-Stark effect. This unique splitting will be intuitively explained in the following paragraphs. (a) Bare states

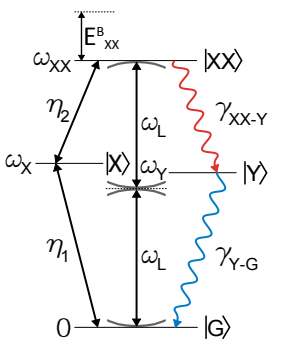

Lab frame (b) Dressed states

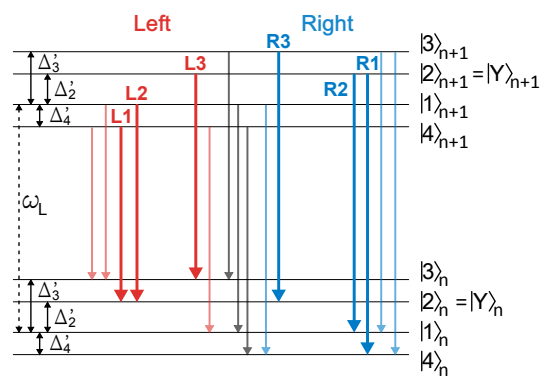

Rotating frame
FIG. 3. (Color online) (a) Energy level diagram of the bare QD states. The $x$-polarized transitions $(|X X\rangle-|X\rangle-|G\rangle)$ are coherently driven by a laser of frequency $\omega_{L}$ with amplitude $\eta_{1,2}$. The biexciton can decay via the $y$-polarized state $|Y\rangle$ by spontaneous emission with rates $\gamma_{X X-Y, Y-G}$ giving rise to the $y$-polarized spectra $S_{Y}$. (b) Dressed energy level diagram and allowed transitions in the rotating frame of the excitation laser $\omega_{L}$. The dressed states are labeled as $|1\rangle,|2\rangle,|3\rangle$ and $|4\rangle$. The $6 y$-polarized transitions, which should be observable in our experiment, are highlighted by bold arrows (Fig.1p). Left (right) denotes the transitions spectrally located to the left or right of the drive laser frequency.

\section{B. Intuitive 4-level atom model}

To develop a simple physical understanding of the measured spectrum, we first describe the system using a driven 4-level simple atom model (Fig. 3(a)). The given states are the biexciton $|X X\rangle$, the $\mathrm{X}$-exciton $|X\rangle$, the Y-exciton $|Y\rangle$ and the ground state $|G\rangle$ with energies $\hbar \omega_{X X}, \hbar \omega_{X}, \hbar \omega_{Y}$ and 0 , respectively. The $X$ - and $Y$ excitons are detuned by a splitting of $\delta_{X Y}=25 \mu \mathrm{eV}$, due to the anisotropic exchange interaction. We analyzed the polarization dependence of the emission emitted to the top, and for this particular QD-cavity system the $x$ and $y$-polarized excitons are almost aligned with the $x$ and $y$-polarized cavity modes (Fig. 11(c)). Thus, the resonant laser coherently drives the $x$-polarized transitions $|X X\rangle-|X\rangle$ and $|X\rangle-|G\rangle$ with Rabi frequency $\eta_{2}$ and $\eta_{1}$, respectively (Fig. 1(d) and Fig. 3(a)). The $y$-polarized transitions are perpendicular to the driving field and do not couple to the light field.

The Hamiltonian of the system in a frame rotating at the laser frequency $\omega_{L}$ is given by

$$
\begin{aligned}
& H=\hbar \Delta_{4}|X X\rangle\left\langle X X\left|+\hbar \Delta_{3}\right| X\right\rangle\left\langle X\left|+\hbar \Delta_{2}\right| Y\right\rangle\langle Y| \\
& +\hbar \eta_{2}\left(\sigma_{X X-X}^{+}+\sigma_{X X-X}^{-}\right)+\hbar \eta_{1}\left(\sigma_{X-G}^{+}+\sigma_{X-G}^{-}\right),
\end{aligned}
$$

where $\sigma_{A-B}^{-}$is the QD lowering operator from state $|A\rangle$ to $|B\rangle$, and $\Delta_{4}=\omega_{X X}-2 \omega_{L}$ and $\Delta_{2,3}=\omega_{Y, X}-\omega_{L}$ are the detunings for the QD states from the drive laser. The time-independent Hamiltonian, under the 2-photon resonance condition $\left(\Delta_{4}=0\right)$, can be represented as a 4 by 4 matrix in terms of bare basis states, namely using 
the $|X X\rangle,|X\rangle,|Y\rangle$ and $|G\rangle$, to obtain

$$
H=\left[\begin{array}{cccc}
0 & \eta_{2} & 0 & 0 \\
\eta_{2} & \Delta_{3} & 0 & \eta_{1} \\
0 & 0 & \Delta_{2} & 0 \\
0 & \eta_{1} & 0 & 0
\end{array}\right]
$$

This Hamiltonian can easily be diagonalized and its 4 eigenvalues are $\Delta_{1,2,3,4}^{\prime}$ with corresponding eigenstates are $|1\rangle,|2\rangle,|3\rangle,|4\rangle$, respectively. Since the $Y$ exciton is not coupled to the drive, it is undressed and represents one of the eigenstates $|2\rangle=|Y\rangle$ with $\Delta_{2}^{\prime}=\Delta_{2}$. The other states $|X X, X, G\rangle$ are dressed by the two simultaneous drives. The $X$-exciton is coupled to the drive; but due to the large detuning of the $x$-polarized transitions $(|X X\rangle-|X\rangle)$ and $(|X\rangle-|G\rangle)$ from the drive laser frequency $\omega_{L}$, the state $|X\rangle$ is minimally dressed and $|3\rangle \approx|X\rangle$ and $\Delta_{3}^{\prime}=\left(\Delta_{3}+\sqrt{\Delta_{3}^{2}+4\left(\eta_{1}^{2}+\eta_{2}^{2}\right)}\right) / 2$. The bare ground $|G\rangle$ and biexciton $|X X\rangle$ states are strongly hybridized to create the new states $|1\rangle$ and $|4\rangle$ with eigenenergies $\Delta_{1,4}^{\prime}=0,\left|\left(\Delta_{3}-\sqrt{\Delta_{3}^{2}+4\left(\eta_{1}^{2}+\eta_{2}^{2}\right)}\right) / 2\right|$. The new eigenstates $|1,4\rangle$ are almost equal admixtures of the ground and the biexciton states.

For a strong drive, multiple manifolds of these new states $(|1,2,3,4\rangle)$ are formed (Fig. 3(b)) separated by drive frequency $\omega_{L}$. These different pump manifolds are identified by a subscript $n$, which represents the number of $x$-polarized photons from the coherent pump. 15 allowed transitions between consecutive pump manifolds determine the total polarization unresolved spectra (see Appendix). Three of them are degenerate at the laser frequency (gray arrows). These are transitions between same states; the transition between state $|Y\rangle$ of two consecutive manifolds is forbidden as it is undressed. The 12 other transitions are symmetric with respect to the laser frequency: 4 are close to the laser frequency, 4 arise from the biexciton emission line and 4 from the exciton emission line. In contrast, in the experiment the exciton and biexciton emission lines split into 2 instead of 4 lines (Fig. 2(c)). To fully understand the experimental results we have to account for the detection scheme, too.

The coherently excited biexciton state $|X X\rangle$ can decay via both excitons states $|X\rangle$ and $|Y\rangle$. As we discussed before, our QD axes are approximately orientated like the cavity axes. If we assume a dipole radiation pattern, illustrated by the colored, vertical sections of a toroid in Fig. 1(e), only the $y$-polarized transitions emit into the side detection path. The x-polarized transitions do not emit parallel to the $x$-axis and with it we cannot detect them using this kind of side detection. Thus, only the $y$-polarized spectrum is measured and the transitions generating the spectra involve the $y$-polarized state $(|2\rangle=|Y\rangle)$, either as initial or as final state. The expected emission spectrum is reduced to 6 spectrally distinct transitions, which are labeled with L1, L2, L3, R3, R2, and R1 in Fig. 3(b). Note that the central transition at $\omega_{L}$ is now missing. The transitions directly indicate

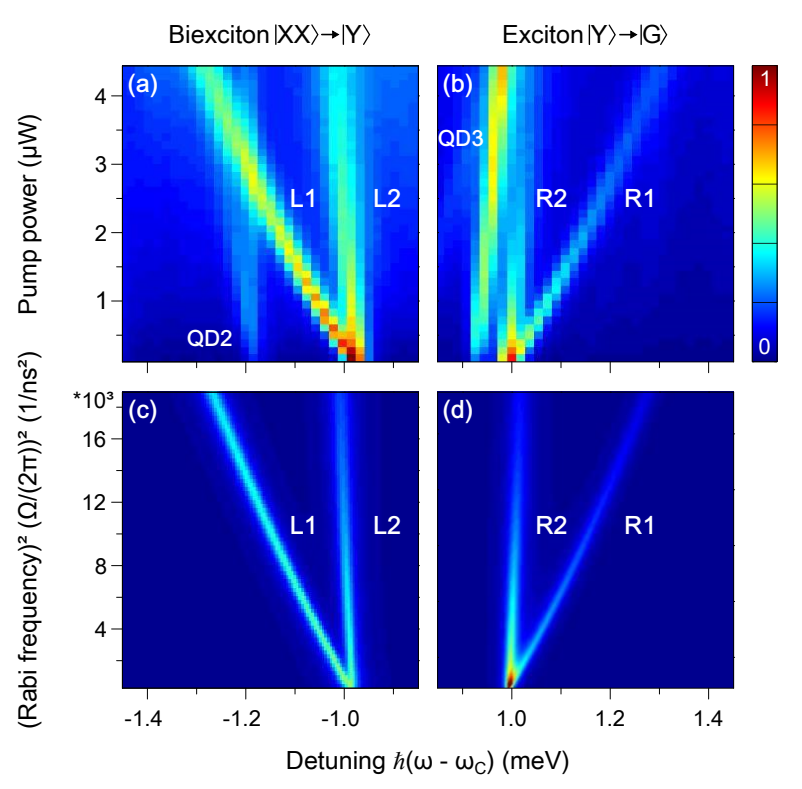

FIG. 4. (Color online) Normalized color-scale maps of $S_{\mathrm{Y}}$ showing dressed transitions around the original biexciton (a) and exciton (b) transitions, for increasing incident pump power. (c) and (d) display the corresponding calculated normalized spectra at $\mathrm{T}=6.8 \mathrm{~K}$. The lines are labeled corresponding to the transitions in Fig. 3(b). Simulations use experimentally determined parameters (see text).

the existence of 3 dressed states $|1,3,4\rangle$ formed due to dressing of the 3 bare states $|G, X, X X\rangle$ by two simultaneous drives. Specifically transitions L3 and R3 near $\omega_{L}$ are transitions between $|Y\rangle$ and $|3\rangle$. This proves that though $|3\rangle$ is similar to the original $|X\rangle$, it is still slightly hybridized with $|X X, G\rangle$. If $|3\rangle=|X\rangle$ this transition would be dipole forbidden.The lines L1, L2, R2, and R1 are the two doublets in Fig. 2(c), emerging out of the initial biexciton and exciton emission lines.

\section{Power and detuning dependent measurements}

Figures 4(a) and (b) display normalized color-scale maps of the PL intensity for $y$-polarized biexciton and exciton transitions, respectively, as a function of emission energy and incident laser power $\mathrm{P}$ of the driving laser. The symmetric splitting $\Delta_{\Omega}$ of the two emission lines shows a linear dependence on the pump power and reaches values of up to $300 \mu \mathrm{eV}$. The labeling of the spectral lines corresponds to the transitions between the dressed states of the dressed 4-level atom as shown in Fig. 3(b) and the splitting $\Delta_{\Omega}$ can be estimated: The frequency of the two right $\mathrm{Y}$ transitions $\left(\mathrm{R}_{2}\right.$ and $\left.\mathrm{R}_{1}\right)$ are $\omega_{L}+\Delta_{2}$ and $\omega_{L}+\Delta_{2}+\Delta_{4}^{\prime}$, respectively, and their splitting $\Delta_{\Omega}=\Delta_{4}^{\prime} \approx \frac{\eta_{1}^{2}+\eta_{2}^{2}}{\Delta_{3}}$ grows linearly with power, at low powers. It is also apparent from the model that lines $\mathrm{L}_{2}$ and $R_{2}$ at transition energies $\omega_{L} \mp \Delta_{2}$ should not move with increasing power as $\Delta_{2}$ is constant. In contrast, the 


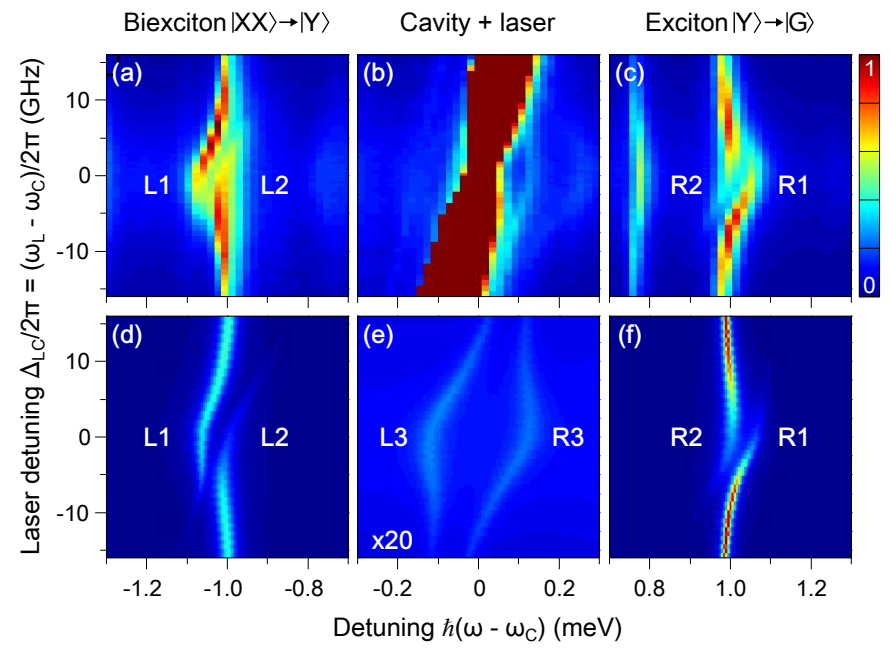

FIG. 5. (Color online) Normalized color-scale map of the PL spectra as a function of energy and laser detuning around the original biexciton (a), cavity (b) and exciton (c) emission lines at a temperature of $20.2 \mathrm{~K}$. The laser itself strongly saturates the scale and also the CCD camera in this experiment. (d) - (f) display the calculated normalized emission spectra. Parameters are the same as in Fig. 4 and incorporate the frequency shifts due to $T=20.2 \mathrm{~K}$ (see text).

experiment reveals also a slight shift of these lines. This effect is not covered by the simple model, but is fully reproduced by the calculated emission spectra in Fig. 4(c, d). We extensively introduce our model including phonon and cavity interactions in Sec. IV.

Figures 5(a)-(c) present $S_{\mathrm{Y}}$ measurements around the original biexciton, cavity, and exciton transitions, respectively, when the frequency of the driving laser is scanned through the cavity resonance with scanning steps of $0.3 \mu \mathrm{eV}(\approx 0.73 \mathrm{GHz})$ at fixed pump power of $6.6 \mu \mathrm{W}$. Note, during several cooling and heating cycles some strain or field changes in the micropillar caused a blueshift of the QD emission with respect to the cavity mode. Therefore, the following experiments were performed at a temperature of $20.2 \mathrm{~K}$ to tune the $\mathrm{QD}$ emission and to almost re-establish the two-photon resonance condition. Hence, the emission lines are slightly shifted to lower energies compared to the previously presented measurements. Figures 5(d)-(f) show again the respective normalized spectra calculations. By tuning the laser frequency through the cavity we observe an anticrossing like behavior for both the exciton and the biexciton emission lines at the same time. The on-resonance doublets (Fig. 5 (a, c) ) correspond to distinct dressed transitions in Fig. 4(a, b). The cavity not only aids field enhancement but also acts as spectral filter for the pump laser. Thus, the effective drive amplitude depends on the detuning (see Sec. IV). This becomes noticeable in the re-shaped anticrossing. In Fig. 5(b) we observe several weaker lines located around the cavity frequency. These lines follow the expected trend of the corresponding calculated tran- sitions $L_{3}, R_{3}$ in Fig. 5(e), but we cannot exclude contributions from other QD emission lines, which are shifted by a strong, cavity-enhanced AC Stark effect. The strong scattered laser light prohibits to clarify this with reasonable certainty.

\section{THEORETICAL INVESTIGATION}

\section{A. Hamiltonian}

In this section we introduce the full system Hamiltonian including acoustic phonons and cavity interactions which is used for the theoretical calculation of $y$-polarized spectra $S_{\mathrm{Y}}$ in Fig. 4 and Fig. 5 . The complete system Hamiltonian in the rotating frame of the drive laser, is given by

$$
\begin{aligned}
& H=\hbar \Delta_{4}|X X\rangle\left\langle X X\left|+\hbar \Delta_{3}\right| X\right\rangle\left\langle X\left|+\hbar \Delta_{2}\right| Y\right\rangle\langle Y| \\
& +\sum_{P=x, y} \hbar \Delta_{c L}^{P} a_{P}^{\dagger} a_{P}+\sum_{q} \hbar \omega_{q} b_{q}^{\dagger} b_{q}+\hbar \Omega\left(a_{x}^{\dagger}+a_{x}\right) \\
& +\hbar g_{2}^{x}\left(\sigma_{X X-X}^{+} a_{x}+a_{x}^{\dagger} \sigma_{X X-X}^{-}\right)+\hbar g_{1}^{x}\left(\sigma_{X-G}^{+} a_{x}+a_{x}^{\dagger} \sigma_{X-G}^{-}\right) \\
& +\hbar g_{2}^{y}\left(\sigma_{X X-Y}^{+} a_{y}+a_{y}^{\dagger} \sigma_{X X-Y}^{-}\right)+\hbar g_{1}^{y}\left(\sigma_{Y-G}^{+} a_{y}+a_{y}^{\dagger} \sigma_{Y-G}^{-}\right) \\
& +\sum_{S=X, Y, X X}|S\rangle\langle S| \sum_{q} \hbar \lambda_{q}^{S}\left(b_{q}^{\dagger}+b_{q}\right),
\end{aligned}
$$

where the $x, y$ polarized cavity modes of frequency $\omega_{c}^{x, y}$ respectively are described by lowering operators $a_{x, y}$ and phonon modes $(q)$ are described by lowering operator $b_{q}$. The four QD transitions are coupled to the two cavities with the coupling $g_{1,2}^{x, y}$, where the superscript describes photon polarization and the subscripts 2 and 1 denote biexciton-to-exciton and exciton-to-ground state transition, respectively. To be consistent with the experiment, the $x$-polarized cavity is driven by an $x$-polarized strong coherent laser with Rabi frequency $\Omega$ and central frequency $\omega_{L}$ and the cavity detunings in the rotating frame is $\Delta_{c L}^{x, y}=\omega_{c}^{x, y}-\omega_{L}$. The last three terms in $H$ account for electron-phonon interactions. Phonon effects are included in the system Hamiltonian using the independent

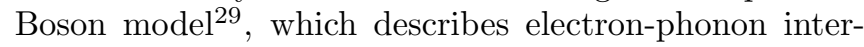
action with the exciton and the biexciton state. The coupling constants $\lambda_{q}^{X, Y, X X}$ couple the $x$-polarized, $y$ polarized exciton and biexciton states respectively with the $q^{\text {th }}$ acoustic phonon mode. The strongest effect of phonons in the QDs under consideration is due to deformation potential coupling 30 to longitudinal acoustic (LA) phonon modes. For such interaction, we take $\lambda_{q}$ as real and following Ref. 31, we assume $\lambda_{q}^{X X}=2 \lambda_{q}^{X}=2 \lambda_{q}^{Y}$ for an ideal QD.

A large detuning between the coherently driven $x$-cavity and the coupled transitions (i.e. $\left.\left|\left(\omega_{X X}-\omega_{X}\right)-\omega_{c}^{x},\left(\omega_{X}-\omega_{G}\right)-\omega_{c}^{x}\right| \approx 1 \mathrm{meV}\right)$ allows us to use an adiabatic approximation where the cavity mode operator $a_{x}$ is replaced by a complex ("c") number $\alpha=\frac{\Omega}{\frac{\kappa_{x}}{2}+i \Delta_{c L}^{x}} \sqrt{32}$, where $\kappa_{x}$ denotes the damping 
rate of the $X$-cavity. The expression follows from the Heisenberg equations for lowering operator $a_{x}$ given by $\frac{d a_{x}}{d t}=\Omega-\left(i \Delta_{c L}^{x}+\frac{\kappa_{x}}{2}\right) a_{x}-i g_{1}^{x} \sigma_{X-G}^{-}-i g_{2}^{x} \sigma_{X X-X}^{-}$(from Eq. (4). In the presence of a strong drive, large detuning and weak coupling, the two QD coupling terms can be neglected and $\alpha$ is the steady-state solution for $a_{x}$. Substituting $\alpha$ for $a_{x}$ in Eq. 4 reduces the Hamiltonian $\mathrm{H}$ to

$$
\begin{aligned}
& H=\hbar \Delta_{4}|X X\rangle\left\langle X X\left|+\hbar \Delta_{3}\right| X\right\rangle\left\langle X\left|+\hbar \Delta_{2}\right| Y\right\rangle\langle Y| \\
& +\hbar \Delta_{c L}^{y} a_{y}^{\dagger} a_{y}+\sum_{q} \hbar \omega_{q} b_{q}^{\dagger} b_{q} \\
& +\hbar \eta_{2}\left(\sigma_{X X-X}^{+}+\sigma_{X X-X}^{-}\right)+\hbar \eta_{1}\left(\sigma_{X-G}^{+}+\sigma_{X-G}^{-}\right) \\
& +\hbar g_{2}^{y}\left(\sigma_{X X-Y}^{+} a_{y}+a_{y}^{\dagger} \sigma_{X X-Y}^{-}\right)+\hbar g_{1}^{y}\left(\sigma_{Y-G}^{+} a_{y}+a_{y}^{\dagger} \sigma_{Y-G}^{-}\right) \\
& +\sum_{S=X, Y, X X}|S\rangle\langle S| \sum_{q} \hbar \lambda_{q}^{S}\left(b_{q}^{\dagger}+b_{q}\right) .
\end{aligned}
$$

Hence the adiabatic elimination of the $X$-cavity reduces the problem to the case of a directly driven biexciton system, where the $x$-polarized transitions $|X X\rangle-|X\rangle$ and $|X\rangle-|G\rangle$ are driven by effective Rabi pump fields $\eta_{2}$ and $\eta_{1}$ respectively, where $\eta_{1,2}=g_{1,2}^{x} \alpha$. The $X$-cavity thus acts as an effective Lorentzian filter for the cavity drive $\Omega$, due to presence of the detuning $\Delta_{c L}^{x}$ in the denominator of $\alpha$. Additional coupling of the QD to the cavity ${ }^{32}$ can be accounted for by redefining the radiative decay rates to include cavity coupling if appropriate (i.e., through the Purcell effect $)^{33}$, but this is not necessary in this large detuning regime. The $y$-polarized cavity is included exactly in the model (Eq. 5). Though the $y$-polarized cavity is not directly driven, it still strongly assists the radiative decay of the QD using $y$-polarized transitions, especially in presence of phonons.

\section{B. Polaron master equation}

To solve for the dynamics of the coupled QD-cavity system in presence of phonons, we sebsequently derive a polaron master equation for the reduced density matrix of the QD-cavity system. We perform a polaron transform 29134 on $H$ (Eq. 5 given by $H^{\prime} \rightarrow e^{P} H e^{-P}$, where $P=|X\rangle\left\langle X\left|\sum_{q} \frac{\lambda_{q}^{X}}{\omega_{q}}\left(b_{q}^{\dagger}-b_{q}\right)+\right| Y\right\rangle\langle Y| \sum_{q} \frac{\lambda_{q}^{Y}}{\omega_{q}}\left(b_{q}^{\dagger}-\right.$ $\left.b_{q}\right)+|X X\rangle\langle X X| \sum_{q} \frac{\lambda_{q}^{X X}}{\omega_{q}}\left(b_{q}^{\dagger}-b_{q}\right)^{29}$. Assuming an ideal $\mathrm{QD} \lambda_{q}^{X X}=2 \lambda_{q}^{X}=2 \lambda_{q}^{Y}=2 \lambda_{q}$ gives a polaron trans- formed Hamiltonian ${ }^{34} H^{\prime}=H_{\mathrm{S}}^{\prime}+H_{\mathrm{B}}^{\prime}+H_{\mathrm{I}}^{\prime}$ where

$$
\begin{aligned}
& H_{\mathrm{S}}^{\prime}=\hbar\left(\Delta_{4}-2 \Delta_{P}\right)|X X\rangle\left\langle X X\left|+\hbar\left(\Delta_{3}-\Delta_{P}\right)\right| X\right\rangle\langle X| \\
& +\left(\Delta_{2}-\Delta_{P}\right)|Y\rangle\langle Y|+\hbar \Delta_{c L}^{y} a_{y}^{\dagger} a_{y} \\
& +\hbar \eta_{2}^{\prime}\left(\sigma_{X X-X}^{+}+\sigma_{X X-X}^{-}\right)+\hbar \eta_{1}^{\prime}\left(\sigma_{X-G}^{+}+\sigma_{X-G}^{-}\right) \\
& +\hbar g_{2}^{\prime y}\left(\sigma_{X X-Y}^{+} a_{y}+a_{y}^{\dagger} \sigma_{X X-Y}^{-}\right)+\hbar g_{1}^{\prime y}\left(\sigma_{Y-G}^{+} a_{y}+a_{y}^{\dagger} \sigma_{Y-G}^{-}\right),
\end{aligned}
$$

where $H_{\mathrm{I}}^{\prime}=X_{g} \zeta_{g}+X_{u} \zeta_{u}$ and $H_{\mathrm{B}}^{\prime}=\sum_{q} \hbar \omega_{q} b_{q}^{\dagger} b_{q}$. The phonon renormalize the field couplings, so that $g_{1,2}^{\prime y}=$ $\langle B\rangle g_{1,2}^{y}, \eta_{1,2}^{\prime}=\langle B\rangle \eta_{1,2}$, and $\langle B\rangle=\left\langle B_{+}\right\rangle=\left\langle B_{-}\right\rangle$, where $B_{ \pm}=\exp \left[ \pm \sum_{q} \frac{\lambda_{q}}{\omega_{q}}\left(b_{q}-b_{q}^{\dagger}\right)\right]$ are the coherent phonon bath displacement operators 29 . The interaction terms ${ }^{34} X_{g}=\hbar \eta_{2}\left(\sigma_{X X-X}^{+}+\sigma_{X X-X}^{-}\right)+\hbar \eta_{1}\left(\sigma_{X-G}^{+}+\sigma_{X-G}^{-}\right)+$ $\hbar g_{2}^{y}\left(\sigma_{X X-Y}^{+} a_{y}+a_{y}^{\dagger} \sigma_{X X-Y}^{-}\right)+\hbar g_{1}^{y}\left(\sigma_{Y-G}^{+} a_{y}+a_{y}^{\dagger} \sigma_{Y-G}^{-}\right)$and $X_{u}=i \hbar \eta_{2}\left(\sigma_{X X-X}^{+}-\sigma_{X X-X}^{-}\right)+i \hbar \eta_{1}\left(\sigma_{X-G}^{+}-\sigma_{X-G}^{-}\right)+$ $i \hbar g_{2}^{y}\left(\sigma_{X X-Y}^{+} a_{y}-a_{y}^{\dagger} \sigma_{X X-Y}^{-}\right)+i \hbar g_{1}^{y}\left(\sigma_{Y-G}^{+} a_{y}-a_{y}^{\dagger} \sigma_{Y-G}^{-}\right)$and the phonon fluctuation operators are $\zeta_{g}=\frac{1}{2}\left(B_{+}+B_{-}-\right.$ $2\langle B\rangle)$ and $\zeta_{u}=\frac{1}{2 i}\left(B_{+}-B_{-}\right)^{34}$. A small polaron shift $\Delta_{P}=\Sigma_{q} \frac{\lambda_{q}^{2}}{\omega_{q}}$ also appears due to the polaron transform and below we will assume this factor is absorbed in the polaron-shifted frequency of the exciton and biexciton states $\left(\Delta_{2,3,4}\right)$. In the continuum limit, the phonon coupling is determined by the phonon spectral function $J_{\mathrm{pn}}(\omega)^{34}$ and we use the continuous form of the phonon spectral function, $J_{\mathrm{pn}}(\omega)=\alpha_{p} \omega^{3} \exp \left[-\frac{\omega^{2}}{2 \omega_{b}^{2}}\right]$, for LA phonon interaction where $\alpha_{p}$ is the exciton-phonon coupling strength and $\omega_{b}$ is the phonon cutoff frequency 3 .

Using Eq. 6, we can next derive a time-local master equation for the reduced density matrix $\rho$ of the QDcavity system; following Ref. 34, we use a second-order Born approximation to obtain the polaron master equation in the interaction picture,

$$
\frac{\partial \tilde{\rho}}{\partial t}=-\frac{1}{\hbar^{2}} \int_{0}^{t} d t^{\prime} \operatorname{Tr}_{\mathrm{B}}\left\{\left[\tilde{H}_{\mathrm{I}}^{\prime}(t),\left[\tilde{H}_{\mathrm{I}}^{\prime}\left(t^{\prime}\right), \tilde{\rho}(t) \rho_{\mathrm{B}}\right]\right]\right\},
$$

where $\tilde{H}_{\mathrm{I}}^{\prime}(t)=e^{i\left(H_{\mathrm{S}}^{\prime}+H_{\mathrm{B}}^{\prime}\right) t / \hbar} H_{\mathrm{I}}^{\prime} e^{-i\left(H_{\mathrm{S}}^{\prime}+H_{\mathrm{B}}^{\prime}\right) t / \hbar}$ and tilde denotes the interaction picture. The operator $\operatorname{Tr}_{\mathrm{B}}$ denotes trace over the phonon bath $\left(\rho_{\mathrm{B}}\right)$ and we assume the full density operator to be separable at all times, through $\rho \rho_{\mathrm{B}} \sqrt{34}$. Performing the trace and transforming back into the Schrödinger picture, we obtain the final form of the polaron master equation 34 , 


$$
\begin{aligned}
& \frac{d \rho}{d t}=\frac{1}{i \hbar}\left[H_{\mathrm{S}}^{\prime}, \rho\right]+\frac{\gamma_{X X-X}}{2} L\left(\sigma_{X X-X}^{-}\right)+\frac{\gamma_{X-G}}{2} L\left(\sigma_{X-G}^{-}\right)+\frac{\gamma_{X X-Y}}{2} L\left(\sigma_{X X-Y}^{-}\right)+\frac{\gamma_{Y-G}}{2} L\left(\sigma_{Y-G}^{-}\right)+\frac{\kappa_{y}}{2} L\left(a_{y}\right) \\
& +\frac{\gamma_{X X-X}^{\prime}}{2} L\left(\sigma_{X X-X}^{+} \sigma_{X X-X}^{-}\right)+\frac{\gamma_{X-G}^{\prime}}{2} L\left(\sigma_{X-G}^{+} \sigma_{X-G}^{-}\right)+\frac{\gamma_{X X-Y}^{\prime}}{2} L\left(\sigma_{X X-Y}^{+} \sigma_{X X-Y}^{-}\right)+\frac{\gamma_{Y-G}^{\prime}}{2} L\left(\sigma_{Y G}^{+} \sigma_{Y G}^{-}\right) \\
& \quad-\frac{1}{\hbar^{2}} \int_{0}^{\infty} d \tau \sum_{m=g, u}\left(G_{m}(\tau)\left[X_{m}, e^{-i H_{S}^{\prime} \tau / \hbar} X_{m} e^{i H_{S}^{\prime} \tau / \hbar} \rho(t)\right]+\text { H.c. }\right),
\end{aligned}
$$

where $G_{g}=\langle B\rangle^{2}(\cosh (\phi(t))-1)$ and $G_{u}=$ $\langle B\rangle^{2} \sinh (\phi(t))$ are the polaron Green functions ${ }^{34}$ and $\langle B\rangle=e^{-\phi(0) / 2}$, where $\phi(t)=$ $\int_{0}^{\infty} d \omega \frac{J_{\mathrm{pn}}(\omega)}{\omega^{2}}\left[\operatorname{coth}\left(\frac{\hbar \omega}{2 k_{B} T}\right) \cos (\omega t)-i \sin (\omega t)\right]$ is the IBM phase function $\frac{34}{3}$; note we have extended the upper limit of the integral in (7) to $t \rightarrow \infty$ to obtain a Markov form which has been shown to be valid ${ }^{34}$. It should be noted that additional incoherent processes like spontaneous emission, pure dephasing and cavity damping are also included in the master equation using respective Lindblad terms where $\kappa_{y}$ is Y-cavity decay rate, $\gamma_{A-B}$ is the spontaneous decay rate between states $|A\rangle$ to $|B\rangle$ and $\gamma_{A-B}^{\prime}$ is pure dephasing rate for the corresponding transition. For the Born-Markov approximation to be valid, in the polaron frame, the system dynamics should be much slower compared to the rate of relaxation of the phonon bath $(\approx 2 \mathrm{ps})$, which is certainly the case of the present study.

The master equation is numerically solved using the quantum optics toolbox $x^{36}$. The simulation parameters used in Fig. 4 and Fig. 5 closely correspond to the measured values and are cavity damping $\hbar \kappa_{x, y}=74 \mu \mathrm{eV}$, $132 \mu \mathrm{eV}\left(\kappa_{x, y} /(2 \pi)=17.9 \mathrm{GHz}, 31.9 \mathrm{GHz}\right)$, excitoncavity coupling $\hbar g_{1}^{x, y}=26.7 \mu \mathrm{eV}$ and biexciton-cavity coupling $\hbar g_{2}^{x, y}=31.0 \mu \mathrm{eV}$. The spontaneous emission (SE) rates are $\gamma_{X-G, Y-G}=1180 \mathrm{ps}^{-1}(\hbar \gamma=$ $0.56 \mu \mathrm{eV})$ and $\gamma_{X X-X, X X-Y}=2^{*} 750 \mathrm{ps}^{-1}(0.44 \mu \mathrm{eV})$. The pure dephasing rate $\gamma_{A B}^{\prime}$, for transition $|A\rangle-|B\rangle$ is $\gamma_{X-G, Y-G, X X-X, X X-Y}^{\prime}=160 \mathrm{ps}^{-1}\left(2 \hbar \gamma^{\prime}=8.2 \mu \mathrm{eV}\right)$. The $x$ - and $y$-polarized exciton and cavity states are detuned by $25 \mu \mathrm{eV}$ and $320 \mu \mathrm{eV}$, respectively. A continuous form of phonon spectral function is used is characterized by electron-phonon coupling strength $\alpha_{P} /(2 \pi)^{2}=0.03$ $\mathrm{ps}^{2}$ and cut-off frequency $\hbar \omega_{b}=0.9 \mathrm{meV}$.

\section{Emission spectrum}

In this section we introduce the formula for the spectrum $S_{\mathrm{Y}}$ plotted in Fig. 4 and Fig. 5 . In the rotating frame, the total spectrum at detector position $\mathbf{r}_{D}$ (with the QD at position $\mathbf{r}_{d}$ ) is given by 3 37/38

$$
\begin{aligned}
& S\left(\mathbf{r}_{D}, \omega\right)=\left\langle\left(\mathbf{E}^{+}\left(\mathbf{r}_{D}, \omega\right)\right)^{\dagger} \mathbf{E}^{+}\left(\mathbf{r}_{D}, \omega\right)\right\rangle= \\
& \lim _{T \rightarrow \infty} \frac{1}{T} \int_{0}^{T} d t \int_{0}^{T} d t^{\prime}\left\langle\mathbf{E}^{-}\left(\mathbf{r}_{D}, t\right) \mathbf{E}^{+}\left(\mathbf{r}_{D}, t^{\prime}\right)\right\rangle e^{i\left(\omega_{L}-\omega\right)\left(t-t^{\prime}\right)},
\end{aligned}
$$

with the electric-field operator defined through $\mathbf{E}^{+}\left(\mathbf{r}_{D}, \omega\right)=\frac{1}{\epsilon_{0}} \sum_{n} \mathbf{G}\left(\mathbf{r}_{D}, \mathbf{r}_{d}, \omega\right) \cdot \mathbf{d}_{n} \sigma_{n}^{-}$; here $\mathbf{G}$ is the photon Green function, and $\mathbf{d}_{n}$ is the dipole moment of the various $n$ transitions aligned along $\hat{x}$ or $\hat{y}$ axes. Assuming a spectrally flat photon reservoir function (as appropriate for emission through radiation, non-cavity, modes), and using the quantum regression theorem, we obtain the total incoherent spectrum from a CW drive,

$$
\begin{aligned}
& S_{\mathrm{Tot}}\left(\mathbf{r}_{D}, \omega\right)=F\left(\mathbf{r}_{D}\right) \lim _{t \rightarrow \infty} \operatorname{Re}\left[\int_{0}^{\infty} d \tau\right. \\
& \left.\quad \sum_{i=X, Y}\left(\left\langle\sigma_{i}^{+}(t+\tau) \sigma_{i}^{-}(t)\right\rangle-\left\langle\sigma_{i}^{+}(t) \sigma_{i}^{-}(t)\right\rangle\right) e^{i\left(\omega_{L}-\omega\right) \tau}\right] \\
& \quad \equiv F\left(\mathbf{r}_{D}\right) S_{\operatorname{Tot}}(\omega),
\end{aligned}
$$

where $\sigma_{X}^{-}=d_{X X-X} \sigma_{X X-X}^{-}+d_{X-G} \sigma_{X-G}^{-}, \sigma_{Y}^{-}=$ $d_{X X-Y} \sigma_{X X-Y}^{-}+d_{Y-G} \sigma_{Y-G}^{-}$and $F\left(\mathbf{r}_{D}\right)$ is a geometrical factor. In this expression $d_{A B}$ corresponds to the magnitude of the dipole moment connecting transition between states $|A\rangle$ to $|B\rangle$ and is determined from the bare spontaneous emission rate connecting these states, i.e., $d_{A B} \propto \sqrt{\gamma_{A B}}$. If one detects only the $y$-polarized emission, then the $y$-detected incoherent spectrum is obtained from

$$
\begin{aligned}
S_{Y}(\omega) & =\lim _{t \rightarrow \infty} \operatorname{Re}\left[\int _ { 0 } ^ { \infty } d \tau \left(\left\langle\sigma_{Y}^{+}(t+\tau) \sigma_{Y}^{-}(t)\right\rangle\right.\right. \\
& \left.\left.-\left\langle\sigma_{Y}^{+}(t) \sigma_{Y}^{-}(t)\right\rangle\right) e^{i\left(\omega_{L}-\omega\right) \tau}\right]
\end{aligned}
$$

\section{Influence of phonon interactions}

Phonon interactions and incoherent losses determine the linewidth and the oscillator strength of the different spectral transitions, as can be observed by comparing an experimental measurement against theoretical calculations with and without phonons in Fig. 6. Without phonon coupling, the oscillator strength of the transitions to the right of the laser (cavity) are relatively stronger 


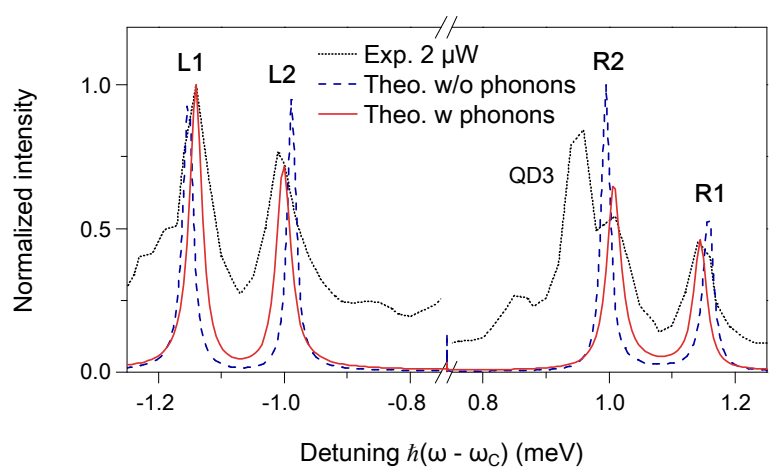

FIG. 6. (Color online) Comparison of experimental spectrum (black, dotted) and the theoretical calculations with (red, solid) and without (blue, dashed) acoustic phonon contributions.

(causing $R 2 \approx L 2, L 1$ ). With phonon interactions, the oscillator strengths of the right transitions (R2) reduce, as observed in Fig. 4(a, b) and Fig. 6. The modified oscillator strength with phonons can be qualitatively understood from the asymmetric phonon-dressed Mollow spectra emitted from single excitons; due to phonon-induced excitation processes, transitions to the left of the pump laser are always fed more strongly than transitions to the right. This is because at low temperatures, phonon emission is more probable than absorption. Coupling to the Y-cavity further enhances this effect. Transitions located to the right of the Y-cavity (higher energy) can feed it more strongly compared to the left ones, through phonon-mediated processes ${ }^{34}$ at low temperatures. The right transitions are hence weaker due to faster phononmediated decay 34 .

Phonon fluctuations are also responsible for observed systematic broadening of the spectral lines and this effect increases with drive strength $\Omega$, due to the presence of the coherent drive terms $\eta_{1,2}$ in $X_{m}$ (Eq. 8), an effect known as excitation induced dephasing. The line splittings $\Delta_{\Omega}$ are also reduced due to the coherent phonon renormalization term $\langle B\rangle$. The linewidth increases roughly as square of the drive power and reaches values of about $40 \mu \mathrm{eV}$. The experimentally observed linewidths are slightly broader as we do not account for the instrument response function in our theory.

\section{E. Mean photon number inside the cavity}

Using the atomic model (Eq. 1), the mean number of $X$-cavity photons in the resonant condition, corresponding to a given line splitting $\Delta_{\Omega}$ can be determined analytically using $N_{c}=\left\langle a^{\dagger} a\right\rangle_{X} \approx \frac{\Delta_{\Omega}\left(\Delta_{\Omega}+\Delta_{3}\right)}{\left(\left(g_{1}^{x}\right)^{2}+\left(g_{2}^{x}\right)^{2}\right)}$. If the phonon fluctuation terms and coupling to the $Y$-cavity is ignored and only coherent phonon effects are considered, then Eq. 6 reduces to the case of a coherently driven biexciton (Eq. 1) and the mean $X$-cavity photon num- ber for a given dressed state splitting under the resonant 2 photon pumping condition can be estimated as $N_{c}=\frac{\Delta_{\Omega}\left(\Delta_{\Omega}+\Delta_{3}\right)}{\langle B\rangle^{2}\left(\left(g_{1}^{x}\right)^{2}+\left(g_{2}^{x}\right)^{2}\right)}$. As $\langle B\rangle \leq 1$ for $T>0$, a larger photon number is required to obtain the same splitting at higher temperatures in the presence of phonons. For Fig. 5 . $\left(\hbar \Delta_{\Omega}=65 \mu \mathrm{eV}, T=20.2 \mathrm{~K}\right)$ we calculate $N_{c} \approx$ 49. This small photon number underlines the importance of the electric field enhancement inside the cavity. In the steady-state the number of photons that enter the cavity equals the number of photons that leave the cavity: $d N_{c} / d t=0=\eta_{c} \eta_{\text {obj }} P_{\text {inc }}-N_{c} \kappa$, with the incident laser power $P_{\text {inc }}$, the coupling efficiency of the driving laser into the cavity mode $\eta_{c}$, the objectives transmission $\eta_{\text {obj }}=0.7$, and the cavity decay rate $\kappa$. Thus, we determine the coupling efficiency to roughly $26 \%$.

\section{PERSPECTIVE APPLICATIONS}

Due to the easy controllable spectral tunability of dressed states via laser excitation power, they resemble a versatile two-photon source operating in the continuous regime. In a very recent work, C. Sanchez Munoz et al ${ }^{39}$ suggest to use a dressed biexciton system for the generation of different non-classical light states, from antibunched and bunched photon pairs with polarization orthogonal to the driving field, to polarization entangled two-photon emission. We anticipate that the emitted photons will show a high degree of indistinguishability similar to the photon emission of Mollow triplet sidebands ${ }^{40}$. This characteristic feature would make them very attractive for many quantum information technologies such as quantum key distribution, quantum teleportation or quantum repeaters. In this work, we intended to demonstrate the possibility to dress three QD states at the same time. The yield of QD-cavity systems which show this particular behavior is rather low (1 pillar out of 15 ). With the perspective of quantum information applications, the sample fabrication can be performed with the purpose of obtaining cavities with specific properties, i.e., the cavity resonance at the twophoton resonance condition. A deterministic fabrication technique would be the most effective way to obtain this goa 41 .

\section{CONCLUSIONS}

In conclusion, we have demonstrated a unique dressed biexciton-exciton system by cavity-enhanced two-photon CW excitation of a semiconductor QD. The dressed states are studied by $\mu \mathrm{PL}$ spectroscopy and reveal a characteristic emission pattern of four distinct emission lines. The splitting of the biexciton-exciton $(|X X\rangle-|Y\rangle)$ and exciton-ground state $(|Y\rangle-|G\rangle)$ transition show linear dependence on the applied power of the driving laser field. We introduced a dressed four-level system to describe and calculate the measurements theoretically, and we have 


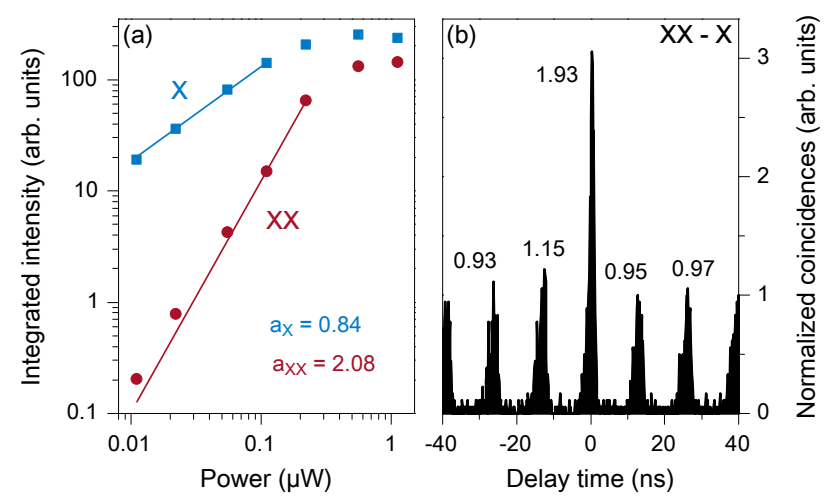

FIG. 7. (Color online). (a) Integrated intensity of the biexciton (circles) and the exciton (squares) emission as a function of the laser power for continuous wave excitation above the GaAs band edge. (b) Second-order cross-correlation measurement under pulsed, resonant two-photon excitation between the two emission lines confirms the cascaded photon emission. The integrated coincidences are given for each peak.

also highlighted the importance of electron-phonon scattering to the model to match more quantitatively using a polaron master equation approach.

\section{ACKNOWLEDGMENTS}

Note: After this paper was submitted, we became aware of a related work on the $\operatorname{arXiv} \underline{42}$.

We thank M. Emmerling for expert sample fabrication. We acknowledge financial support of the Deutsche Forschungsgemeinschaft (DFG) within the SFB/TRR21 and the projects MI500/23-1 and Ka2318/4-1, and the Natural Sciences and Engineering Research Council of Canada.

\section{Appendix A: Biexciton-exciton cascade}

We demonstrate that the investigated emission line pair belongs to a biexciton-exciton cascade. Therefore, we plot the integrated intensities for continuous wave excitation above the GaAs band edge as a function of the incident laser power (Fig. 7(a)). The slopes of 0.84 for the exciton and 2.08 for the biexciton emission are typical values for InAs/GaAs QDs.

An intensity cross-correlation measurement between the two emission lines serves as a reliable proof of the cascade. Therefore, we spatially split the collected emission with a beam splitter and use two monochromators to spectrally separate the lines. Two avalanche photo diodes serve as detectors. The second-order cross-correlation measurement under resonant two-photon excitation is plotted in Fig. 7 (b). The strong bunching at zero time delay proves the cascaded emission.
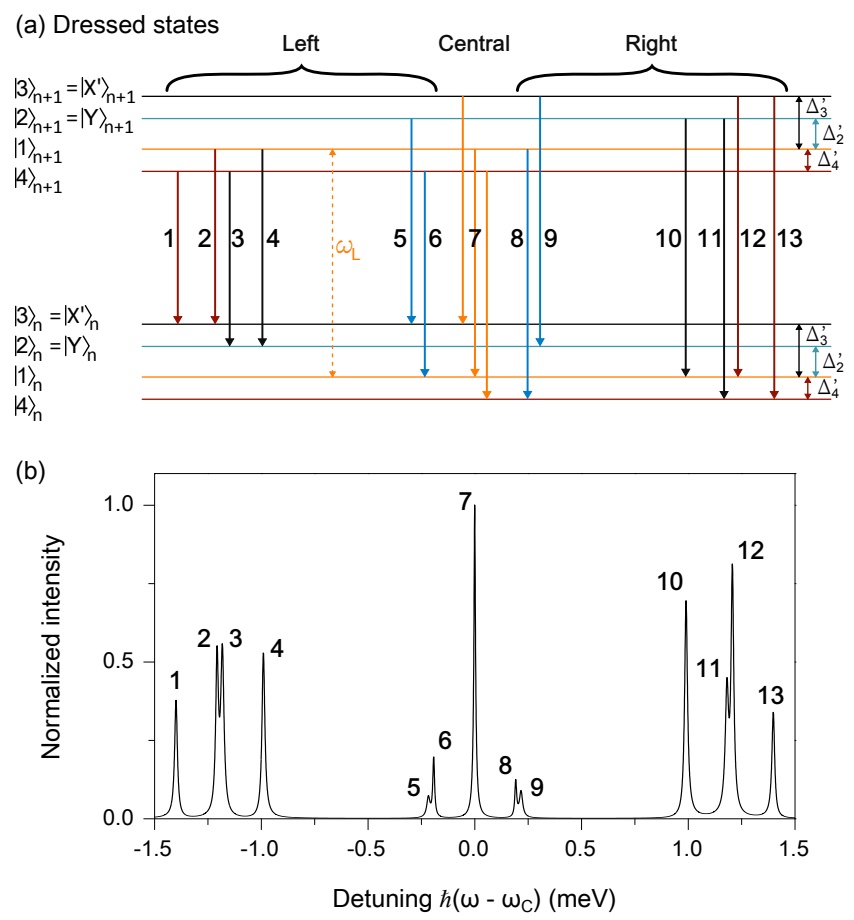

FIG. 8. (Color online). (a) Schematic energy level diagram and transitions between the dressed states of the different pump-induced manifolds. The $x$-polarized transitions involving the state $\left|X^{\prime}\right\rangle(1,2,12,13)$ and giving rise to spectral transitions around the original biexciton $(|X X\rangle-|X\rangle)$ and exciton transitions $(|X\rangle-|G\rangle)$ are marked by light (red) arrows. The $y$-polarized transitions involving the $Y$ exciton $(3,4,10$, 11) and giving rise to spectral transitions around the original biexciton $(|X X\rangle-|Y\rangle)$ and exciton transitions $(|Y\rangle-|G\rangle)$ are marked by dark (black) arrows. The transitions $(5,6$, $8,9)$ are spectrally closer to the laser drive frequency. Note that level $|3\rangle$ is also marked as $\left|X^{\prime}\right\rangle$ to indicate its similarity with state $|X\rangle$ (see text). (b) Calculated emission spectrum $S_{\text {Tot }}$ without phonons showing the different transitions in (a). Simulation parameters are the same as in Fig. 4.

\section{Appendix B: Complete set of dressed states}

In this appendix, we show the complete set of transitions (including both $x$ - and $y$-polarized photons) between the dressed states (Fig. 3(b)) in Fig. 8(a). The transitions at the drive frequency $\omega_{L}$ in Fig. 8 (a) are marked 'central' and the symmetric transitions in frequency, about the drive are marked 'left' and 'right'. In Fig. 8(b) we show the complete polarization unresolved spectra $S_{\text {Tot }}$ and identify the spectral lines corresponding to these transitions. In the current numbering scheme, transitions $(3,4,5,9,10,11)$ correspond to lines (L1, L2, L3, R3, R2, R1) of the $y$-polarized spectra $S_{Y}$ shown in Fig. 3(b). The current calculations ingores effects of the $y$-cavity coupling and phonon interactions to avoid additional effects of broadening. 
* f.hargart@ihfg.uni-stuttgart.de

† kroy@physics.queensu.ca

1 B. R. Mollow, Phys. Rev. 188, 1969 (1969)

2 C. Cohen-Tannoudji, J. Dupont-Roc, and G. Grynberg, Atom-Photon Interactions (WILEY-VCH, 1998).

3 X. Xu, B. Sun, P. R. Berman, D. G. Steel, A. S. Bracker, D. Gammon, and L. J. Sham, Science 317, 929 (2007).

${ }^{4}$ G. Jundt, L. Robledo, A. Högele, S. Fält, and A. Imamoğlu, Phys. Rev. Lett. 100, 177401 (2008).

5 B. D. Gerardot, D. Brunner, P. A. Dalgarno, K. Karrai, A. Badolato, P. M. Petroff, and R. J. Warburton, New Journal of Physics 11, 013028 (2009)

${ }^{\circ}$ A. Muller, W. Fang, J. Lawall, and G. S. Solomon, Phys. Rev. Lett. 101, 027401 (2008)

E. B. Flagg, A. Muller, J. W. Robertson, S. Founta, D. G. Deppe, M. Xiao, W. Ma, G. J. Salamo, and C. K. Shih, Nature Phys. 5, 203 (2009)

8 A. N. Vamivakas, Y. Zhao, C.-Y. Lu, and M. Atatüre, Nature Phys. 5, 198 (2009)

9 S. Ates, S. M. Ulrich, S. Reitzenstein, A. Löffler, A. Forchel, and P. Michler, Phys. Rev. Lett. 103, 167402 (2009)

${ }^{10}$ Y.-M. He, Y. He, Y.-J. Wei, D. Wu, M. Atature, C. Schneider, S. Hofling, M. Kamp, C.-Y. Lu, and J.-W. Pan, Nature Nanotech. 8, 213 (2013).

it A. Ulhaq, S. Weiler, S. M. Ulrich, R. Roßbach, M. Jetter, and P. Michler, Nature Photon. 6, 238 (2012)

12 S. M. Ulrich, S. Ates, S. Reitzenstein, A. Löffler, A. Forchel, and P. Michler, Phys. Rev. Lett. 106, 247402 (2011)

${ }^{13}$ C. Roy and S. Hughes, Phys. Rev. Lett. 106, 247403 (2011)

${ }^{14}$ H. Kim, T. C. Shen, K. Roy-Choudhury, G. S. Solomon, and E. Waks, Phys. Rev. Lett. 113, 027403 (2014).

15 Y.-J. Wei, Y. He, Y.-M. He, C.-Y. Lu, J.-W. Pan, C. Schneider, M. Kamp, S. Höfling, D. P. S. McCutcheon, and A. Nazir, Phys. Rev. Lett. 113, 097401 (2014)

16 R.-C. Ge, S. Weiler, A. Ulhaq, S. M. Ulrich, M. Jetter, P. Michler, and S. Hughes, Opt. Lett. 38, 1691 (2013).

17 Y. He, Y.-M. He, J. Liu, Y.-J. Wei, H. Y. Ramírez, M. Atatüre, C. Schneider, M. Kamp, S. Höfling, C.-Y. Lu, and J.-W. Pan, Phys. Rev. Lett. 114, 097402 (2015).

18 M. Peiris, B. Petrak, K. Konthasinghe, Y. Yu, Z. C. Niu, and A. Muller, Phys. Rev. B 91, 195125 (2015)

19 R. Bose, D. Sridharan, G. S. Solomon, and E. Waks, Appl. Phys. Lett. 98, 121109 (2011)

${ }_{20}$ P. M. Vora, A. S. Bracker, S. G. Carter, T. M. Sweeney, M. Kim, C. S. Kim, L. Yang, P. G. Brereton, S. E. Economou, and D. Gammon, Nat Commun 6, (2015)

21 B. Gayral, J. M. Gérard, B. Legrand, E. Costard, and V. Thierry-Mieg, Appl. Phys. Lett. 72, 1421 (1998)
${ }^{22}$ K. Brunner, G. Abstreiter, G. Böhm, G. Tränkle, and G. Weimann, Phys. Rev. Lett. 73, 1138 (1994).

23 T. Flissikowski, A. Betke, I. A. Akimov, and F. Henneberger, Phys. Rev. Lett. 92, 227401 (2004).

24 S. Stufler, P. Machnikowski, P. Ester, M. Bichler, V. M. Axt, T. Kuhn, and A. Zrenner, Phys. Rev. B 73, 125304 (2006)

25 H. Jayakumar, A. Predojevic, T. Huber, T. Kauten, G. S. Solomon, and G. Weihs, Phys. Rev. Lett. 110, 135505 (2013)

${ }^{20}$ M. Müller, S. Bounouar, K. D. Jöns, M. Glässl, and P. Michler, Nature Photon. 8, 224 (2014)

27 P.-L. Ardelt, L. Hanschke, K. A. Fischer, K. Müller, A. Kleinkauf, M. Koller, A. Bechtold, T. Simmet, J. Wierzbowski, H. Riedl, G. Abstreiter, and J. J. Finley, Phys. Rev. B 90, 241404 (2014).

28 S. Bounouar, M. Müller, A. M. Barth, M. Glässl, V. M. Axt, and P. Michler, Phys. Rev. B 91, 161302 (2015).

29 I. Wilson-Rae and A. Imamoğlu, Phys. Rev. B 65, 235311 (2002)

so B. Krummheuer, V. M. Axt, and T. Kuhn, Phys. Rev. B 65, $195313(2002)$.

${ }^{31}$ U. Hohenester, G. Pfanner, and M. Seliger, Phys. Rev. Lett. 99, 047402 (2007)

32 S. Hughes and H. J. Carmichael, New Journal of Physics 15, 053039 (2013)

33 E. M. Purcell, Phys. Rev. 69, 681 (1946)

34 C. Roy and S. Hughes, Phys. Rev. B 85, 115309 (2012).

35 S. Weiler, A. Ulhaq, S. M. Ulrich, D. Richter, M. Jetter, P. Michler, C. Roy, and S. Hughes, Phys. Rev. B 86, 241304 (2012).

36 S. M. Tan, J. Opt. B: Quant. Semiclass. Opt. 1, 424 (1999)

37 H. T. Dung, L. Knöll, and D.-G. Welsch, Phys. Rev. A 66, $063810(2002)$

38 P. Yao, C. Van Vlack, A. Reza, M. Patterson, M. M. Dignam, and S. Hughes, Phys. Rev. B 80, 195106 (2009).

39 C. Sánchez Muñoz, F. P. Laussy, C. Tejedor, and E. del Valle, ArXiv e-prints (2015), arXiv:1506.05050 [quant-ph]

40 S. Weiler, D. Stojanovic, S. M. Ulrich, M. Jetter, and P. Michler, Phys. Rev. B 87, 241302 (2013)

41 A. Dousse, L. Lanco, J. Suffczyński, E. Semenova, A. Miard, A. Lemaître, I. Sagnes, C. Roblin, J. Bloch, and P. Senellart, Phys. Rev. Lett. 101, 267404 (2008).

42 P.-L. Ardelt, M. Koller, T. Simmet, L. Hanschke, A. Bechtold, A. Regler, J. Wierzbowski, H. Riedl, K. Müller, and J. J. Finley, ArXiv e-prints (2015), arXiv:1511.00503 [cond-mat.mes-hall] 